\title{
Assessment of the usefulness of skin autofluorescence as an indicator of disease activity and of the risk of developing diabetes in patients suffering from rheumatoid arthritis
}

\author{
Paweł Żuchowski ${ }^{1}$, Katarzyna Kolossa², Sławomir Jeka ${ }^{2,3}$, Rafał Wojciechowski², \\ Arnika Wilińska-Jankowska², Marzena Waszczak ${ }^{4}$ \\ ${ }^{1}$ Independent Section of Scientific Research Coordination, J. Biziel University Hospital No. 2, Bydgoszcz, Poland \\ ${ }^{2}$ Clinic of Rheumatology and Systemic Connective Tissue Disorders, J. Biziel University Hospital No. 2, Bydgoszcz, Poland \\ ${ }^{3}$ Ludwik Rydygier Collegium Medicum in Bydgoszcz, UMK in Torun, Poland \\ ${ }^{4}$ Centre for Clinical Trials, Warsaw, Poland
}

\begin{abstract}
Objectives: Diabetes develops much more often in patients suffering from rheumatoid arthritis (RA) than in healthy population. One of the parameters which allow to evaluate the risk of developing diabetes and cardiovascular diseases (CVD) is the level of advanced glycation end products (AGE) in the skin. In patients suffering from RA, an increase in AGE level may be also linked with the course of the underlying disease. The aim of the study was to evaluate the correlation between the AGE level and the course of RA as well as other risk factors for the development of diabetes and CVD. Material and methods: The study included 148 patients divided into three groups: group I - patients with RA ( $n=102,79 \mathrm{~F} / 23 \mathrm{M})$, group II - patients with RA and diabetes $(n=21,14 \mathrm{~F} / 7 \mathrm{M})$, group III healthy individuals $(n=25,16 \mathrm{~F} / 9 \mathrm{M})$. Each patient underwent a skin autofluorescence signal (SAF) examination with an AGE Reader, which allows the assessment of AGE level, as well as being subjected to the laboratory tests panel. Additionally, patients from group I and II have had their DAS28 (ESR) indicator calculated.

Results: In groups I, II, and III, the respective mean SAF values, expressed in arbitrary units [au], were to $2.54,2.74$, and $1.96 \mathrm{au}$. Between-group differences in terms of mean SAF values were statistically significant $(p<0.05)$.

Conclusions: Significantly higher mean SAF values in groups I and II as compared to group III suggest that the increase in the AGE level in patients with RA is linked with the underlying disease and does not have to correspond with the real risk of diabetes and CVD. In conclusion, despite the known limitations of the technique, measuring AGE levels allows for closer monitoring of RA patients who are at a higher risk of developing diabetes.
\end{abstract}

Key words: diabetes, rheumatoid arthritis, autofluorescence.

\section{Introduction}

Rheumatoid arthritis (RA) is a chronic systemic immune-mediated connective tissue disease. RA may be accompanied by other conditions, such as diabetes or cardiovascular disease (CVD). CVD is the main cause of death in RA patients [1]. Patients with RA often develop coronary artery disease and experience myocardial infarctions, cerebral thromboembolic events, and sudden deaths.

Address for correspondence:

Paweł Żuchowski, Samodzielna Sekcja ds. Badań Naukowych, J. Biziel University Hospital No. 2, Bydgoszcz, Poland,

e-mail: p.zuchowski81@gmail.com

Submitted: 26.10.2015; Accepted: 7.11.2015 
The risk of cardiovascular complications is highest in patients over 40 years of age and is higher in males than in females [1]. Disorders of glucose metabolism are also a relatively common complication of RA. They constitute a serious therapeutic problem, which significantly affects both the course of RA and its treatment. Disturbances of glucose metabolism in RA may occur as clinically manifest diabetes, impaired glucose tolerance or insulin resistance, each increasing the risk of CVD [2]. The prevalence of diabetes in RA patients is approximately $13-16 \%$ [3]. It means that the incidence of diabetes in RA patients is three times higher than in the general population [3]. RA may be accompanied by several glucose metabolism disturbances, including type 1 diabetes, type 2 diabetes, and latent autoimmune diabetes of adults (LADA). Higher incidence rates of type 1 diabetes in patients with RA are most likely associated with the presence of particular HLA class II genes responsible for specific autoimmune disorders [4]; autoimmune polyglandular syndromes are an example of such disorders. The prevalence of particular types of diabetes in RA patients is difficult to determine due to diagnostic problems with differentiating between type 2 diabetes and LADA [5].

The detection of anti-GAD antibodies plays a key role in the differential diagnosis. This is important because anti-GAD-positive patients should start their insulin treatment early. The presence of anti-GAD antibodies may be affected by medications, decreasing the sensitivity and specificity of these assessments. Methotrexate (MTX) treatment in RA patients with concomitant LADA leads to lowered anti-GAD levels [6]. Thus, if antibody testing for diabetes in this group of patients is performed late, it becomes virtually impossible to accurately diagnose LADA. Consequently, these patients may be misdiagnosed as having type 2 diabetes, which results in inappropriate treatment with oral hypoglycemic agents [5]. One study found no apparent signs or symptoms of diabetes in $20 \%$ of subjects [7].

Patients with no clinical manifestations of diabetes tend to develop chronic complications already in the early stages of the disease, as their insulin resistance increases. These are mainly cardiovascular complications due to atherosclerosis [8]. Since patients with RA are also at risk for cardiovascular complications, they should undergo regular fasting blood glucose tests, or, alternatively, they should have their 2-hour glucose tolerance level tested (75 g OGTT). These are the simplest tests and are recommended by both the World Health Organization (WHO) and the American Diabetes Association (ADA) [5].

However, as hypoglycemia is a late sign of diabetes, treatment is often initiated relatively late. Therefore, al- ternative diagnostic methods are used, such as the hyperinsulinemic-euglycemic clamp technique for evaluating insulin resistance, as well as assessments of fasting insulin, proinsulin-like component, $\mathrm{HbA1c}$, or adiponectin levels. The risk of developing diabetes and CVD can also be evaluated in vivo with fluorescence spectroscopy, an alternative diagnostic technique which is currently used with increasing frequency in various medical specialties [9-11]. This type of assessment can be conducted with an AGE Reader, which helps determine the risk of diabetes based on skin auto-fluorescence (SAF) analysis [12]. This examination assesses advanced glycation end products (AGES), which are a heterogeneous group of molecules formed in non-enzymatic reactions of reducing sugars, ascorbic acid, and other hydrocarbons containing amine acid, fats, or nucleic acids. Reactions of this type also occur in healthy individuals during the natural ageing process. However, in patients with diabetes and CVD these reactions are significantly accelerated. Animal studies demonstrated that AGE levels increase significantly within a few weeks after the onset of diabetes. AGEs are deposited mainly in the kidneys, blood vessels, and skin [13].

Currently, the AGE Reader (Diagnoptics; the Netherlands) is the only available spectrometer allowing for AGE level assessment. The use of this spectrometer is limited to measuring AGE concentration in the skin. This is a non-invasive method based on ultraviolet (UVA) light absorbed by AGE molecules in the skin. Subsequently, the photons initially absorbed by the AGEs are emitted in a process called auto-fluorescence, and are counted by the built-in photomultiplier. This helps determine the intensity of the fluorescence signal for the wavelengths corresponding to AGE-emitted photons.

\section{Purpose of the study}

The purpose of this study was: 1) to assess the usefulness of assessing skin AGE levels via the non-invasive SAF method in detecting glucose metabolism disturbances in RA patients; and 2) to determine the correlation between AGE levels and RA activity measured with the disease activity score DAS28.

\section{Material and methods}

A total of 148 subjects undergoing diagnostic evaluation or treatment at the Rheumatology Clinic were included in this study. The study lasted from the beginning of April 2014 until the end of June 2015. The subjects were divided into three groups: group I - patients with RA and no evidence of diabetes of any type; group IIpatients with RA and type 2 diabetes; group III - individuals in whom both systemic connective tissue diseases 
Table I. Basic demographic characteristics in the study groups

\begin{tabular}{|c|c|c|c|c|}
\hline \multirow[t]{2}{*}{ Group } & \multirow[t]{2}{*}{ Number of subjects } & \multicolumn{2}{|c|}{ Gender } & \multirow{2}{*}{$\begin{array}{c}\text { Mean age } \\
\text { (median; SD) }\end{array}$} \\
\hline & & $\mathrm{F}$ & $M$ & \\
\hline I & 102 & 79 & 23 & $\begin{array}{c}51.5 \\
(52 \pm 12.97)\end{array}$ \\
\hline ॥ & 21 & 14 & 7 & $\begin{array}{c}51.9 \\
(50 \pm 10.54)\end{array}$ \\
\hline III & 25 & 16 & 9 & $\begin{array}{c}48.8 \\
(48 \pm 8.95)\end{array}$ \\
\hline
\end{tabular}

and diabetes mellitus were excluded. None of the patients from groups I and II were treated with glucocorticosteroids. Table I shows basic demographic characteristics in each study group.

RA was diagnosed in group I and group || subjects based on 2010 American College of Rheumatology (ACR)/ European League Against Rheumatism (EULAR) criteria. Group III subjects constituted a control group. The only study exclusion criteria were skin conditions and scars or tattoos present on the palmar aspect of both forearms, as well as excessive suntan and the use of bronzers or other sunless tanning products. Such alterations of the skin would preclude a reliable assessment with an AGE Reader. Study subjects, irrespective of their study group, underwent the following assessments: SAF; lipid panel, including total cholesterol, triglycerides (TG), high-density lipoproteins (HDL), low-density lipoproteins (LDL); fasting blood glucose; HbA1c; ESR; and their body mass index (BMI) was calculated based on their height and weight.

Groups I and II were also evaluated for DAS28 (ESR).

Skin fluorescence was measured using the AGE Reader SU, capable of assessing AGE concentration in the skin. The device was connected to a computer and operated via the appropriate software. Figure 1 shows a sample result obtained with an AGE Reader.

Based on the SAF value, four risk groups for diabetes and CVD were identified:

- no risk - the measured SAF value was less than or equal to the mean value for the given age range. In the case of individuals over 70 years old, the only factor increasing the risk of diabetes and CVD was age itself,

- low risk - the measured SAF value was greater than the mean value for the given age range, but did not exceed 1 standard deviation (SD),

- increased risk - the measured SAF value exceeded the mean value by more than 1 SD, but it was less than 2.9. In such cases, other parameters associated with diabetes and CVD should also be assessed,

- high risk - the measured SAF value was greater than or equal to 2.9. High risk patients must be referred for additional evaluations.
The chi-square test was used to assess the correlation between the tested parameters and the Mann-Whitney $U$ test and Student's $t$ test was used for normally distributed data to compare the results obtained in the study groups. Any $p$ value of $\leq 0.1$ was considered to be statistically significant. SD and median values were also calculated for some of the data collected.

\section{Results}

Mean SAF values in both groups of subjects with RA were similar, but they differed significantly from the mean value in the control group, which is presented in Fig. 1. The groups did not differ significantly in terms of mean age. This is important, as the SAF value increases with age, which is shown in Fig. 2. This proves that the observed differences in mean SAF values were actually a result of the health-related differences in study group subjects and not the differences in mean age between study groups.

The two RA study groups showed no significant differences with regard to any of the laboratory test results.

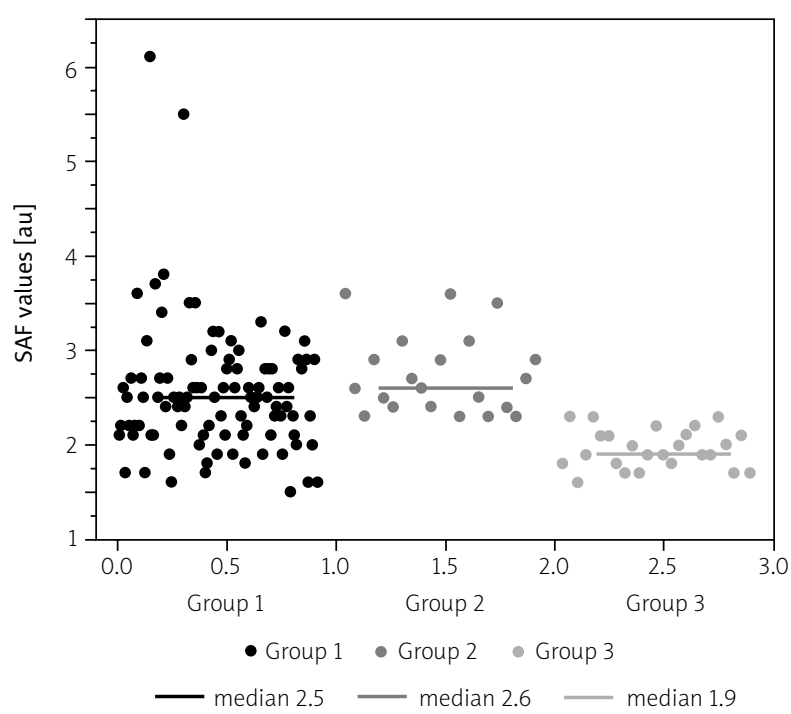

Fig. 1. SAF values in study groups. 


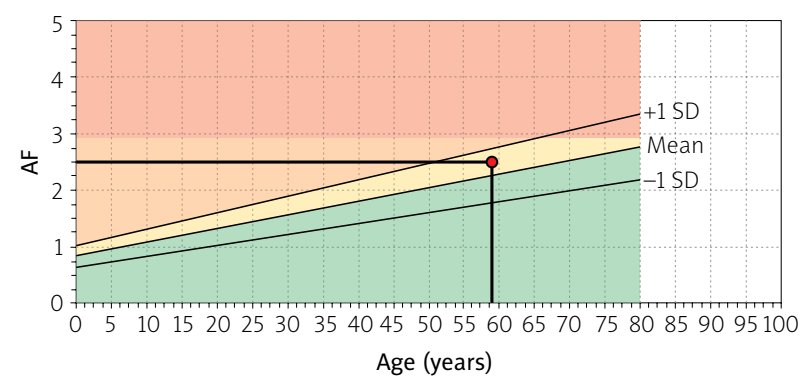

Normal Group: No CV risk _ Risk Group II: Increased CV risk

Risk Group I: Limited increase _ Risk Group III: Definite CV risk of $\mathrm{CV}$ risk

Fig. 2. A sample result obtained with an AGE Reader.

However, both RA study groups differed significantly from the control group in mean values of most laboratory parameters (Table II). Also in the second group of patients with RA and diabetes, BMI was higher than in the other groups, which is not surprising. But it was not a statistically significant difference.

No correlation was observed between the DAS28 value and the risk of diabetes and CVD in patients with RA.

Group I showed a correlation between ESR and the risk of developing diabetes and CVD $(p<0.05$, chisquare test). The lack of such correlation in the other groups was associated with a considerably wide distribution of ESR results and small group size.

Mean SAF values were much higher in groups I and II in comparison to those in group III, suggesting that the increase in AGE concentration in RA patients is associated with the underlying disease and does not necessarily reflect the actual risk of diabetes and CVD. This finding is consistent with other reports stating that the increase in AGE concentration in RA is due to oxidative stress [14]. Consequently, the predictive value of this examination in determining the risk of diabetes and CVD may be low.

Mean disease activity in study subjects with RA was relatively low, which might explain the lack of correlation between AGE concentrations and DAS28 values. This is consistent with conclusions by Groot et al. [13].

Based on this study, the existence of a correlation between SAF and DAS28 values cannot be excluded. What is needed is a study including a group of patients whose disease activity (and the corresponding DAS28 score) is much higher.

In addition, we found no correlation between SAF and DAS28 values, which may be due to the fact that the total score is considerably affected by the number of tender joints, and the latter does not necessarily reflect the activity of the inflammatory process. Tender joints also include joints with irreversible destructive changes, where active synovitis is no longer present. Therefore, future studies should focus on determining whether there is a correlation between the number of swollen joints (which is a much better indicator of disease activity) and SAF.

One interesting aspect to develop in this study would be to look for a possible correlation between SAF values and the SAS 1 score that illustrates current trends in RA activity evaluation [15].

\section{Discussion}

Measuring the AGE concentration is known to present a considerable challenge despite the fact that an increase in AGE concentration with age and in various dis-

Table II. Overview of patient examination data in each study group and the comparison of statistical significance of inter-group differences

\begin{tabular}{|c|c|c|c|c|c|c|}
\hline & \multicolumn{3}{|c|}{ Group } & \multicolumn{3}{|c|}{$p$-value } \\
\hline & I & II & III & I-II & I-III & $\|-I I \mid$ \\
\hline $\mathrm{SAF}($ mean $\pm \mathrm{SD})$ & $2.54 \pm 0.68$ & $2.74 \pm 0.43$ & $1.96 \pm 0.21$ & - & 0.089 & 0.036 \\
\hline $\mathrm{BMI}($ mean $\pm \mathrm{SD})$ & $25.5 \pm 4.22$ & $26.92 \pm 4.52$ & $24.9 \pm 3.97$ & - & - & - \\
\hline $\begin{array}{l}\text { Total cholesterol } \\
(\text { mean } \pm \text { SD })[\mathrm{mg} / \mathrm{dl}]\end{array}$ & $209.91 \pm 44.92$ & $202.38 \pm 45.95$ & $175.16 \pm 18.57$ & - & 0.012 & 0.000 \\
\hline $\mathrm{HDL}($ mean $\pm \mathrm{SD})[\mathrm{mg} / \mathrm{dl}]$ & $60.52 \pm 14.1$ & $57.48 \pm 17.52$ & $84.08 \pm 18.74$ & - & 0.000 & 0.000 \\
\hline $\mathrm{LDL}($ mean $\pm \mathrm{SD})[\mathrm{mg} / \mathrm{dl}]$ & $137.7 \pm 38.63$ & $141.38 \pm 60.16$ & $118.48 \pm 30.31$ & - & 0.022 & 0.046 \\
\hline $\mathrm{TG}($ mean $\pm \mathrm{SD})[\mathrm{mg} / \mathrm{dl}]$ & $110.54 \pm 59.84$ & $135.05 \pm 60.16$ & $94.72 \pm 23.44$ & - & - & 0.003 \\
\hline $\mathrm{ESR}(\mathrm{mean} \pm \mathrm{SD})[\mathrm{mm} / \mathrm{h}]$ & $20.84 \pm 16.19$ & $24.09 \pm 16.65$ & $12.76 \pm 4.99$ & - & 0.015 & 0.002 \\
\hline $\mathrm{HbA1C}($ mean $\pm \mathrm{SD})[\%]$ & $5.5 \pm 0.31$ & $5.6 \pm 0.43$ & $5.29 \pm 0.24$ & - & 0.002 & 0.003 \\
\hline $\begin{array}{l}\text { Fasting blood glucose } \\
\text { (mean } \pm \mathrm{SD})[\mathrm{mg} / \mathrm{dl}]\end{array}$ & $92.77 \pm 12.28$ & $94.19 \pm 12.85$ & $92.44 \pm 11.53$ & - & - & - \\
\hline DAS 28 (ESR) (mean) & 2.89 & 3.01 & - & - & - & - \\
\hline
\end{tabular}


orders has been well documented. Currently, the most common techniques include high-performance liquid chromatography (HPLC), enzyme-linked immunosorbent assay (ELISA), and immunohistochemical staining methods [13]. The greatest problem is the comparison of results from various laboratories [13]. Moreover, due to the heterogeneity of AGEs it is sometimes difficult to determine whether their concentrations are actually associated with the underlying disease. Today, AGE concentration is measured mainly in research facilities, and there are no generally available and approved techniques or kits for AGE assessment [13]. The lack of standardized measurement techniques leads to problems in interpreting results obtained by different research teams. Fluorescence spectroscopy appears to be an alternative assessment method. However, the development of techniques for reliable assessment of AGE concentration in various tissues may prove extremely difficult, because the phenomenon of auto-fluorescence exists in tissues and may cause the fluorescence of AGEs to be drowned by auto-fluorescence of other molecules. However, one unquestionable advantage of this assessment technique is that it helps identify patients who are at risk of developing diabetes and CVD over the next few years. This is especially true of patients with other chronic conditions, such as RA, which are associated with a higher risk of diabetes and CVD.

\section{Conclusions}

Despite the known limitations of the technique, measuring AGE concentrations allows for closer monitoring of RA patients who are at higher risk of developing diabetes. This assessment, in combination with regular standard diagnostic tests for diabetes, may help to diagnose diabetes much earlier, institute appropriate treatment, and limit the risk of complications, including cardiovascular ones.

At the current stage of research it could be very valuable to begin the AGE level after the diagnosis of RA, and during subsequent visits, when the indicators of disease activity such as DAS28, the Clinical Disease Activity Index (CDAI) and the Simplified Disease Activity Index (SDAI) are evaluated. This would enable monitoring of SAF changes in the patients during the treatment and determination of the correlation of SAF with changes in disease activity and duration of RA. Therefore, it would enable evaluation of the utility of SAF as a test to mon- itor the effectiveness of treatment and the prognostic value.

The authors declare no conflict of interest.

\section{References}

1. Olas J, Gajos G, Bucka J. Cardiovascular mortality in rheumatoid arthritis patients. Post Dermatol Alergol 2006; 23: 26-30.

2. Cojocaru M, Cojocaru IM, Silosi J, Vrabied CD. Metabolic syndrome in rheumatoid arthritis. Maedica (Buchar) 2012; 7: 148-152.

3. Walewska E, Rupiński R, Filipowicz-Sosnowska A. Zaburzenia metabolizmu glukozy u chorych na reumatoidalne zapalenie stawów. Reumatologia 2008; 46: 356-360.

4. Simard JF, Mittleman MA. Prevalent rheumatoid arthritis and diabetes among NHANES III participants aged 60 and older. J Rheumatol 2007; 34: 469-473.

5. Doran M. Rheumatoid arthritis and diabetes mellitus: evidence for an association? J Rheumatol 2007; 34: 460-462.

6. van Deutekom AW, Nurmohamed MT, Peters MJ, et al. Methotrexate and its effect on the anti-GAD titre in two patients with rheumatoid arthritis and diabetes mellitus. Ann Reum Dis 2008; 67: 1051-1052.

7. Sieradzki J, Grzeszczak W, Wójcikowski C i wsp. oraz grupa badaczy SCREEN-POL. Czynniki ryzyka i objawy cukrzycy a występowanie cukrzycy w badaniach SCREEN-POL. Diabetol Pol 1999; 6 supl. 2: S212-S223.

8. Després JP, Lamarche B, Mauriège $P$, et al. Hyperinsulinemia as an independent risk factor for ischaemic heart disease. N Engl J Med 1996; 334: 952-957.

9. Szczęsny W, Żuchowski P, Fisz JJ, et al. The ultrastructure of rectus sheath in patients with inguinal hernias and healthly controls - an evaluation by fluorescent spectroscopy and microspectrometric techniques. Hernia 2009; 13: 64-65.

10. Chwirot B, Chwirot S, Sypniewska N, et al. Fluorescence in situ detection of human cutaneous melanoma: study of diagnostic parameters of the method. J Invest Dermatol 2001; 117: 1449-1451.

11. Jeka S, Prochorec-Sobieszek M. Zastosowanie spektroskopii fluorescencyjnej w diagnostyce amyloidozy - doniesienie wstępne. Reumatologia 2010; 48: 230-236.

12. Stirban A, Heinemann L. Skin autofluorescence - a non-invasive measurement for assessing cardiovascular risk and risk of diabetes. European Endocrinology 2014; 10: 106-110.

13. de Groot L, Hinkema H, Westra, et al. Advanced glycation end products are increased in rheumatoid arthritis patients with controlled diseases. Arthritis Res Ther 2011; 13: R205.

14. Kulbacka J, Saczko J, Chwitkowska A. Oxidative stress in cells damage processes. Pol Merkur Lekarski 2009; 27: 44-47.

15. Grassi W, Gaywood I, Pande I, et al. From DAS 28 to SAS 1. Clin Exp Rheumatol 2012; 30: 649-651. 